\title{
Hydrodynamics of polar liquid crystals
}

\author{
William Kung* and M. Cristina Marchetti \\ Department of Physics, Syracuse University, Syracuse, New York 13244, USA \\ Karl Saunders \\ Department of Physics, California Polytechnic State University, San Luis Obispo, California 93407, USA
}

(Received 25 October 2005; revised manuscript received 4 January 2006; published 30 March 2006)

\begin{abstract}
Starting from a microscopic definition of an alignment vector proportional to the polarization, we discuss the hydrodynamics of polar liquid crystals with local $C_{\infty_{v}}$ symmetry. The free energy for polar liquid crystals differs from that of nematic liquid crystals $\left(D_{\infty h}\right)$ in that it contains terms violating the $\mathbf{n} \rightarrow-\mathbf{n}$ symmetry. First we show that these $\mathcal{Z}_{2}$-odd terms induce a general splay instability of a uniform polarized state in a range of parameters. Next we use the general Poisson-bracket formalism to derive the hydrodynamic equations of the system in the polarized state. The structure of the linear hydrodynamic modes confirms the existence of the splay instability.
\end{abstract}

DOI: 10.1103/PhysRevE.73.031708

PACS number(s): 61.30. $-\mathrm{v}, 47.20 .-\mathrm{k}, 03.50 .-\mathrm{z}$

\section{INTRODUCTION}

In the past few decades, our understanding of complex materials has greatly benefited from the increasing sophistication of technology and experimental designs. Depending upon the various properties of the constituent molecules, such as geometric shape, electric and magnetic moments, as well as chemical affinity, we are able to observe a variety of phases beyond the traditional crystalline and liquid phases. Examples range from the traditional nematic and smectic liquid crystal phases [1] to the recently discovered smectic blue phase [2] or the banana phases [3]. The possibility of ferroelectric order in liquid crystals has attracted much attention both from a fundamental and practical viewpoint. Among the commonly observed liquid crystalline phases, only the chiral smectic- $C^{*}$ phase is known to be polar. Ferroelectricity in this phase was discovered in 1975 [4] and the related phenomenon of antiferroelectricity in liquid crystals was discovered in 1989 [5]. In these chiral liquid crystals, polarity is realized via the introduction of chiral carbon atoms into achiral molecules. Ferroelectric switching was also recently confirmed in the banana smectic phases [3] consisting of achiral bent-core molecules. The packing structure in these banana phases induces a polar order in the layers of these liquid-crystal systems.

The possibility of ferroelectric and antiferroelectric order in achiral polar liquid crystals composed of molecules carrying strong permanent electrical dipole moments has been a long-standing theoretical and experimental question. Experimentally, ferroelectric order due to dipole-dipole interactions between rod-like aromatic copolyesters molecules has been reported [6-10] by the Tokyo Tech group. In this case the nematic liquid crystal is biaxial and polarity was observed along both symmetry axes via second-harmonic generation. Analytical (generally mean-field) and numerical calculations [11-16], as well as simulations [17-20], have argued that

*Electronic address: wkung@ physics.syr.edu there is no fundamental reason forbidding the establishment of ferroelectric order in polar liquid crystals, assuming appropriate surface stabilization.

The simplest ferroelectric liquid crystal of this type would be a uniaxial liquid of molecules carrying a permanent electric dipole moment which lies parallel to the molecular axis [21]. In this case ferroelectricity would result from strong coupling among the dipoles (possibly aided by surface effects), yielding an ordered state with all dipoles pointing in the same direction. In their classic textbook de Gennes and Prost argue that such dipolar molecules would always be asymmetric in shape, with head and tails of different size. This would naturally yield a splay deformation, making the liquid ferroelectric state unstable [22]. Theoretical work has indeed shown that any type of molecular asymmetry will suppress ferroelectric order in nematic liquid crystals, possibly replacing it with modulated polar phases [16]. In this paper we show that the instability of an ordered ferroelectric phase of uniaxial polar liquid crystals is more general than originally suggested by De Gennes and Prost. We demonstrate that in uniaxial polar liquid crystals a ferroelectric phase is theoretically possible but only for a limited range of parameters. Outside this range the uniformly polarized state is unstable to splay distortions, regardless of molecular shape. This may explain the difficulties encountered in establishing experimentally the existence of a ferroelectric polar state. We employ a standard Poisson bracket formalism [30] to derive the hydrodynamic equations for a liquid of uniaxial rodlike molecules carrying permanent dipole moments. From these equations we obtain the hydrodynamic modes in the uniformly polarized state and show that in a range of parameters the ordered state becomes unstable against the growth of long-wavelength splay fluctuations. This is consistent with an analysis of the free energy which indicates the presence of a mechanical instability for the same parameter range. Our derivation is based solely on symmetry arguments and makes no assumption on the shape of the molecules.

The familiar nematic liquid crystal phase is characterized by a broken rotational symmetry due to macroscopic order of the rodlike molecules along a fixed direction in space. The 
broken symmetry direction is identified by a unit vector $\mathbf{n}$, known as the director. The states of director $\mathbf{n}$ and $-\mathbf{n}$ are indistinguishable. If the molecules carry a permanent electric dipole moment, in the ordered state there are as many "up" dipoles as "down" dipoles and the system is not ferroelectric on large scales. The main difference between a polar liquid crystal and the familiar nematic phase is the breaking of such a $\mathcal{Z}_{2}$ symmetry $(\mathbf{n} \rightarrow-\mathbf{n})$. If the molecules carry a dipole moment, on average the dipoles are pointing in the same direction in the ordered state and the system can be ferroelectric on macroscopic scales. The order parameter characterizing the transition from the isotropic liquid phase to the polarized phase is the alignment vector $\mathbf{P}$, proportional to the mean polarization of the system. This should be contrasted to the nematic order parameter $Q_{i j}$, which is a rank-two tensor, also known as the alignment tensor. In uniaxial systems the mean values of both the alignment vector and the alignment tensor in an ordered phase can be expressed in terms of a unit vector pointing along the broken symmetry direction

$$
\begin{aligned}
\mathbf{P} & =P_{0} \mathbf{n}, \\
Q_{i j} & =S\left(n_{i}^{\prime} n_{j}^{\prime}-\frac{1}{3} \delta_{i j}\right) .
\end{aligned}
$$

The unit vectors $\mathbf{n}$ and $\mathbf{n}^{\prime}$ are the directors in the polarized and nematic states, respectively. A macroscopic polarized state is characterized by $P_{0} \neq 0$. From this it follows that $S$ $\neq 0$ as well in the polarized state, and $\mathbf{n}^{\prime}=\mathbf{n}$. The nematic phase has $P_{0}=0$, but $S \neq 0$. In the following we derive hydrodynamic equations for a polar liquid crystal. The hydrodynamic fields in the isotropic phase are simply the five conserved densities (number density, momentum density, and energy density). In the polarized phase we must add the two broken symmetry variables corresponding to the two independent components of the director $\mathbf{n}$. In addition, we obtain the dynamical equation for the full alignment vector $\mathbf{P}$, which determines the order parameter for the polarizedisotropic phase transition. Its magnitude is not a hydrodynamic variable, but it plays an important role in describing the dynamics near the transition, where its relaxation rate may become very slow.

Our derivation starts in Sec. II with the microscopic definitions of the relevant hydrodynamic fields. Using standard definitions from classical mechanics, in Sec. III we obtain the coarse-grained Poisson brackets for these macroscopic fields. Careful consideration is given to the derivation of the Poisson bracket between the alignment vector $\mathbf{P}$ and the momentum density $\mathbf{g}$ and that between the director $\mathbf{n}$ and $\mathbf{g}$. A similar approach was taken in recent work on deriving the low-frequency hydrodynamics of nematic liquid crystals and nematic polymers $[23,24]$. In Secs. IV and V, we obtain the dynamical equation for the director $\mathbf{n}$ and the alignment vector $\mathbf{P}$, as well as for the conserved densities. These equations contain new terms violating the $\mathcal{Z}_{2}$ symmetry of the nematic phase. Finally, in Sec. VI we examine the hydrodynamic modes and demonstrate the splay instability induced by the new $\mathcal{Z}_{2}$-odd terms.

\section{DEFINITION OF MICROSCOPIC FIELD VARIABLES}

We start by defining the microscopic quantities of interest. The system is composed of $N$ identical liquid crystal molecules, indexed by $\alpha$. Each molecule consists of $s$ atoms treated as point particles, with $m^{\mu}$ and $q^{\mu}$ the mass and the charge of the $\mu$ th atom, respectively. We denote by $\mathbf{r}^{\alpha \mu}$ the position of the $\mu$ th atom in the $\alpha$ th molecule. The position of the center of mass of the $\alpha$ th molecule is given by

$$
\mathbf{r}^{\alpha}=\frac{\sum_{\mu} m^{\mu} \mathbf{r}^{\alpha \mu}}{\sum_{\mu} m^{\mu}},
$$

and $\Sigma_{\mu} m^{\mu}=m^{0}$ is the molecular mass. We assume that the molecules are neutral,

$$
\sum_{\mu} q^{\mu}=0
$$

but possess a nonvanishing dipole moment $\mathbf{d}^{\alpha}$, given by

$$
\mathbf{d}^{\alpha}=\sum_{\mu} q^{\mu} \mathbf{r}^{\alpha \mu} \equiv d^{\alpha} \hat{\nu}^{\alpha},
$$

where $\hat{\nu}^{\alpha}$ is a unit vector along the molecular axis,

$$
\hat{\nu}^{\alpha}=\frac{\sum_{\mu} q^{\mu} \mathbf{r}^{\alpha \mu}}{\left|\sum_{\mu} q^{\mu} \mathbf{r}^{\alpha \mu}\right|}
$$

and

$$
d^{\alpha}=\hat{\nu}^{\alpha} \cdot \mathbf{d}^{\alpha} .
$$

The microscopic mass density $\hat{\rho}(\mathbf{r})$, momentum density $\hat{\mathbf{g}}(\mathbf{r})$, and dipole moment density $\hat{\mathbf{d}}(\mathbf{r})$ are defined in the usual way as

$$
\begin{gathered}
\hat{\rho}(\mathbf{r})=\sum_{\alpha, \mu} m^{\mu} \delta\left(\mathbf{r}-\mathbf{r}^{\alpha \mu}\right), \\
\hat{\mathbf{g}}(\mathbf{r})=\sum_{\alpha, \mu} \mathbf{p}^{\alpha \mu} \delta\left(\mathbf{r}-\mathbf{r}^{\alpha \mu}\right), \\
\hat{\mathbf{d}}(\mathbf{r})=\sum_{\alpha} \mathbf{d}^{\alpha} \delta\left(\mathbf{r}-\mathbf{r}^{\alpha}\right),
\end{gathered}
$$

where $\mathbf{p}^{\alpha \mu}$ is the momentum of the $\mu$ th atom on the $\alpha$ th molecule. The macroscopic mean variables describing the dynamics of equilibrium fluctuations in the system are obtained from the microscopic ones after coarse graining, $\rho(\mathbf{r})=[\hat{\rho}(\mathbf{r})]_{c}, \mathbf{g}(\mathbf{r})=[\hat{\mathbf{g}}(\mathbf{r})]_{c}, \mathbf{d}(\mathbf{r})=[\hat{\mathbf{d}}(\mathbf{r})]_{c}$, as described, for instance, in Refs. [1,24].

The macroscopic coarse-grained fields, denoted by $\Phi_{a}(\mathbf{r}, t)$, are hydrodynamic or quasihydrodynamical variables whose characteristic decay times are much longer than the underlying microscopic time scales and diverge in the limit of long wavelength. They are either conserved variables or variables associated with broken symmetries of the system. They evolve in time according to $[1,24]$ 


$$
\frac{\partial \Phi_{a}(\mathbf{r}, t)}{\partial t}=V^{a}(\mathbf{r})-\Gamma_{a b} \frac{\partial \mathcal{H}}{\partial \Phi_{b}(\mathbf{r})},
$$

where $\Gamma_{a b}$ is the dissipative tensor, $\mathcal{H}$ is the systems Hamiltonian, and $V^{a}$ is the reactive term or nondissipative velocity given by

$$
V_{a}(\mathbf{r})=-\int d^{3} r^{\prime} P_{a b}\left(\mathbf{r}, \mathbf{r}^{\prime}\right) \frac{\delta \mathcal{H}}{\delta \Phi_{b}\left(\mathbf{r}^{\prime}\right)} .
$$

The Einstein summation convention on repeated indices is understood and

$$
P_{a b}\left(\mathbf{r}, \mathbf{r}^{\prime}\right)=\left\{\Phi_{a}(\mathbf{r}), \Phi_{b}(\mathbf{r})\right\}=-P_{b a}\left(\mathbf{r}^{\prime}, \mathbf{r}\right)
$$

denotes the Poisson bracket of the coarse-grained variables. This is defined as

$$
\left\{\Phi_{a}(\mathbf{r}), \Phi_{b}\left(\mathbf{r}^{\prime}\right)\right\}=\left[\left\{\hat{\Phi}_{a}(\mathbf{r}), \hat{\Phi}_{b}\left(\mathbf{r}^{\prime}\right)\right\}\right]_{c},
$$

with

$$
\left\{A(\mathbf{r}), B\left(\mathbf{r}^{\prime}\right)\right\}=\sum_{\alpha, \mu} \sum_{i}\left(\frac{\partial A(\mathbf{r})}{\partial p_{i}^{\alpha \mu}} \frac{\partial B\left(\mathbf{r}^{\prime}\right)}{\partial r_{i}^{\alpha \mu}}-\frac{\partial A(\mathbf{r})}{\partial r_{i}^{\alpha \mu}} \frac{\partial B\left(\mathbf{r}^{\prime}\right)}{\partial p_{i}^{\alpha \mu}}\right) .
$$

The kinetic tensor $\Gamma_{\mu \nu}$ must be symmetric and $\partial \Phi_{\mu}(\mathbf{r}, \mathbf{t}) / \partial t$ and $\delta \mathcal{H} / \delta \Phi_{\nu}$ must have opposite signs under time reversal. The coarse-grained dynamical equations (11) can be rigorously derived from microscopic principles [25-27].

Polar order is described by the alignment vector $\mathbf{P}(\mathbf{r})$, defined as

$$
\mathbf{P}(\mathbf{r}) \equiv \sum_{\alpha} \hat{\nu}^{\alpha} \delta\left(\mathbf{r}-\mathbf{r}_{\alpha}\right) .
$$

The alignment vector $\mathbf{P}(\mathbf{r})$ embodies the fluctuations in the dipoles' orientation. We also write

$$
\mathbf{P}(\mathbf{r}) \equiv \rho(\mathbf{r}) S(\mathbf{r}) \mathbf{n}(\mathbf{r}) .
$$

The scalar field $S(\mathbf{r})$ encodes information on the degree of local alignment of the dipoles, i.e., $S(\mathbf{r})$ is the order parameter for the isotropic-ferroelectric nematic phase transition. The unit vector $\mathbf{n}(\mathbf{r})$ is the macroscopic director. As previously mentioned, the vectorial nature of $\mathbf{P}(\mathbf{r})$ is in sharp contrast to the tensorial character of the usual nematic order parameter $Q_{i j}(r)$. This mathematical distinction leads to new terms in the free energy of a polar fluid that are not allowed for a nematic liquid crystal.

\section{FREE ENERGY AND STABILITY OF THE POLARIZED PHASE}

Before proceeding with the derivation of the hydrodynamic equations for the polarized state, it is instructive to discuss the coarse-grained free energy of a polar fluid. This is given by

$$
\begin{gathered}
F=F_{p}+F_{n}, \\
F_{p}=\int d^{3} r B \frac{\delta \rho(\mathbf{r})}{\rho_{0}} \nabla \cdot \mathbf{n}(\mathbf{r})+\cdots,
\end{gathered}
$$

$$
\begin{aligned}
F_{n}= & \frac{1}{2} \int d^{3} r\left\{C_{1}\left(\frac{\delta \rho}{\rho_{0}}\right)^{2}+C_{2}(\nabla \rho)^{2}+\cdots\right. \\
& +K_{1}(\boldsymbol{\nabla} \cdot \mathbf{n})^{2}+K_{2}(\mathbf{n} \cdot \boldsymbol{\nabla} \times n)^{2} \\
& \left.+K_{3}(\mathbf{n} \times \boldsymbol{\nabla} \times n)^{2}+\cdots\right\},
\end{aligned}
$$

where $\delta \rho(\mathbf{r})=\rho(\mathbf{r})-\rho_{0}$ is the deviation of the local density from its equilibrium value $\rho_{0}$. The contribution $F_{n}$ is the free energy of a nematic liquid crystal which contains only terms symmetric with respect to $\mathbf{n} \rightarrow-\mathbf{n}$, with $K_{1}, K_{2}$, and $K_{3}$ the usual Frank constants for splay, twist and bend deformations, respectively. The contribution $F_{p}$ contains additional terms that break that symmetry and are only allowed in a fluid that is macroscopically polar. In general a term $\sim \boldsymbol{\nabla} \cdot \mathbf{n}(\mathbf{r})$ is also allowed in the free energy. This will give rise to a surface term that will favor a splay distortion of the molecules [corresponding to a nonzero $\boldsymbol{\nabla} \cdot \mathbf{n}(\mathbf{r})]$. In the absence of surface stabilization, this would always destroy the polar order. In this work we assume that there is sufficient surface stabilization to suppress the surface term and to prevent the associated splay.

In addition to a possible surface instability, it is apparent from the form of the free energy that a polarized liquid crystal can also exhibit a bulk instability to splay deformations. The latter is associated with the term $\sim \delta \rho(\mathbf{r}) \nabla \cdot \mathbf{n}(\mathbf{r})$ that cannot be eliminated by surface stabilization. The existence of this instability can be understood by rewriting the terms in the free energy involving density fluctuations and splay deformations of the director as

$$
\begin{gathered}
\int \frac{d^{3} r}{2}\left\{C_{1}\left(\frac{\delta \rho}{\rho_{0}}\right)^{2}+K_{1}\left(\nabla_{\perp} \cdot \delta \mathbf{n}\right)^{2}+2 B \frac{\delta \rho}{\rho_{0 \perp}} \cdot \delta \mathbf{n}\right\} \\
=\int \frac{d^{3} r}{2}\left\{\frac{C_{1}}{\rho_{0}^{2}}\left[\delta \rho+\frac{B \rho_{0}}{C_{1}} \nabla_{\perp} \cdot \delta \mathbf{n}\right]^{2}\right. \\
\left.+\left[K_{1}-\frac{B^{2}}{C_{1}}\right]\left(\nabla_{\perp} \cdot \delta \mathbf{n}\right)^{2}\right\},
\end{gathered}
$$

where $\boldsymbol{\nabla}=\left(\boldsymbol{\nabla}_{\perp}, \partial_{z}\right)$. It is then clear that the effect of the coupling of density fluctuations to splay yield a downward renormalization of the splay elastic constant $K_{1}$. For $|B|$ $>\sqrt{K_{1} C_{1}}$ splay fluctuations becomes energetically favorable and will clearly destabilize the ordered state. The instability is suppressed if the fluid is incompressible since the renormalization of the Frank constant $K_{1}$ vanishes when $C_{1} \rightarrow \infty$. The stable ground state for $|B|>\sqrt{K_{1} C_{1}}$ is expected to be characterized by director configurations that are spatially inhomogeneous in the $x$ direction, perpendicular to the broken symmetry direction, with associated spatial structures in the density. The precise director configurations will depend on boundary effects, as well as on applied external fields (electric field in the case of ferroelectric order). The dynamics of the polarized state discussed below will of course reflect the existence of this instability, which will lead to the growth of the hydrodynamic mode associated with splay fluctuations for $|B|>\sqrt{K_{1} C_{1}}$. 
In order to examine the possible relevance of the splay instability to experimental systems we need to estimate the various elastic constants. We consider a fluid of rods of linear size $l$ interacting via a short-range repulsive interaction of strength $U$. The rods carry a permanent electric dipole moment of magnitude $d$, which yields a dipolar coupling of strength $U_{D} \sim k_{c} d^{2} / r^{3}$ between two molecules at distance $r$, where $k_{c}$ is the Coulomb's law constant. The bulk compressional modulus $C_{1}$ has dimensions of energy density and its size is controlled by the repulsive part of the interaction, with $C_{1} \sim U / l^{3}$. The splay Frank coefficient has dimensions of energy/length and can be estimated as $K_{1} \sim U / l$. This yields an estimate for the critical value $B_{0}=\sqrt{C_{1} K_{1}}$, where the splay instability occurs: $B_{0} \sim U / l^{2}$. The elastic constant $B$ that couples density and splay fluctuations has dimensions of energy $/(\text { length })^{2}$. Assuming that it is controlled by interactions that favor alignment of the dipoles carried by the liquid crystal molecules, we estimate $B \sim U_{P} / l^{2}$, where $U_{P}$ is the strength of such interaction. The splay instability will then occur for $U_{P} \sim U$. For dipoles at a distance $r \sim \rho_{0}^{-1 / 3}$, a dimensional estimate yields $U_{P} \sim k_{c} d^{2} \rho_{0}$. Taking $\rho_{0}$ $\sim 10^{-27} \mathrm{~m}^{-3}$ and $d \sim 2$ Debye [29] we estimate $U_{P}$ $\sim 2.5 \mathrm{meV}$. This is smaller than $U \sim 0.1 \mathrm{eV}$, suggesting that a stable polarized phase may, in principle, be possible. Of course, if the interaction is purely dipolar, appropriate surface stabilization will also be required, as discussed above.

\section{POISSON BRACKETS FOR A POLAR LIQUID CRYSTAL}

We now return to the derivation of hydrodynamics via the Poisson-bracket method. Using Eq. (15), it is straightforward to evaluate the Poisson-bracket relations between the various hydrodynamic fields. Our goal is to obtain equations describing the dynamics at long wavelengths. We will therefore only keep terms of lower order in the gradients of the hydrodynamic fields and expand the $\delta$ function as

$$
\begin{aligned}
\delta\left(\mathbf{r}-\mathbf{r}^{\alpha \mu}\right)= & \delta\left(\mathbf{r}-\mathbf{r}^{\alpha}-\Delta \mathbf{r}^{\alpha \mu}\right)=\delta\left(\mathbf{r}-\mathbf{r}^{\alpha}\right)-\Delta r_{k}^{\alpha \mu} \nabla_{k} \delta\left(\mathbf{r}-\mathbf{r}^{\alpha}\right) \\
& +O\left(\boldsymbol{\nabla}^{2}\right),
\end{aligned}
$$

where $\Delta \mathbf{r}^{\alpha \mu}=\mathbf{r}^{\alpha \mu}-\mathbf{r}^{\alpha}$. The required nonvanishing Poissonbrackets are

$$
\begin{gathered}
\left\{\rho(\mathbf{r}), g_{i}\left(\mathbf{r}^{\prime}\right)\right\}=\nabla_{i} \delta\left(\mathbf{r}-\mathbf{r}^{\prime}\right) \rho\left(\mathbf{r}^{\prime}\right), \\
\left\{g_{i}(\mathbf{r}), g_{j}\left(\mathbf{r}^{\prime}\right)\right\}=g_{i}\left(\mathbf{r}^{\prime}\right) \nabla_{j} \delta\left(\mathbf{r}-\mathbf{r}^{\prime}\right)-\nabla_{i}^{\prime}\left[\delta\left(\mathbf{r}-\mathbf{r}^{\prime}\right) g_{j}\left(\mathbf{r}^{\prime}\right)\right] \\
\left\{P_{i}(\mathbf{r}), g_{j}\left(\mathbf{r}^{\prime}\right)\right\}=\nabla_{j}\left[P_{i}(\mathbf{r}) \delta\left(\mathbf{r}-\mathbf{r}^{\prime}\right)\right]-\delta_{i j} P_{k}(\mathbf{r}) \nabla_{k} \delta\left(\mathbf{r}-\mathbf{r}^{\prime}\right) \\
+\lambda_{i j k}(\mathbf{r}) \nabla_{k} \delta(\mathbf{r}-\mathbf{r}),
\end{gathered}
$$

where

$$
\hat{\lambda}_{i j k}=\sum_{\alpha} \hat{\nu}_{\mathbf{i}}^{\alpha} \hat{\nu}_{\mathbf{j}}^{\alpha} \hat{\nu}_{\mathbf{k}}^{\alpha} \delta\left(\mathbf{r}-\mathbf{r}_{\alpha}\right)
$$

is a symmetric third-order tensor that depends on the degree of molecular alignment. We assume that its coarse-grained counterpart $\lambda_{i j k}$ depends only on the alignment vector $\mathbf{P}(\mathbf{r})$. Quite generally, $\lambda_{i j k}$ can then be written as

$$
\lambda_{i j k}=\lambda_{1}\left(\delta_{i j} P_{k}+\delta_{j k} P_{i}+\delta_{i k} P_{j}\right)+\lambda_{2} \frac{P_{i} P_{j} P_{k}}{P^{2}},
$$

where $P(\mathbf{r})=\mathbf{n} \cdot \mathbf{P}=\rho S$. The two coefficients $\lambda_{1}$ and $\lambda_{2}$ are not independent. By contracting both sides of Eq. (27) with suitable combinations of $\delta_{i j}$ and $n_{k}$ we obtain

$$
\begin{gathered}
n_{k} \lambda_{i i k}=\rho S=\left(4 \lambda_{1}+\lambda_{2}\right) \rho S, \\
n_{i} n_{j} n_{k} \lambda_{i j k}=\sum_{\alpha}\left(\hat{\nu}_{\alpha} \cdot \mathbf{n}\right)^{3} \delta\left(\mathbf{r}-\mathbf{r}_{\alpha}\right)=\left(3 \lambda_{1}+\lambda_{2}\right) \rho S .
\end{gathered}
$$

The two coefficients can then be expressed in terms of single microscopic quantity, $\lambda$, given by

$$
\lambda=\frac{1}{\rho(\mathbf{r}) S(\mathbf{r})}\left[\sum_{\alpha}\left(\hat{\nu}_{\alpha} \cdot \mathbf{n}\right)^{3} \delta\left(\mathbf{r}-\mathbf{r}_{\alpha}\right)\right]_{c},
$$

as

$$
\begin{aligned}
& \lambda_{1}=\frac{1-\lambda}{2}, \\
& \lambda_{2}=\frac{5 \lambda-3}{2} .
\end{aligned}
$$

Equation (27) can then be rewritten in terms of $\lambda$ as

$$
\lambda_{i j k}=\frac{1-\lambda}{2}\left[\delta_{i j}^{T} P_{k}+\delta_{i k}^{T} P_{j}+\delta_{j k}^{T} P_{i}\right]+\lambda n_{j} n_{k} P_{i},
$$

where $\delta_{i j}^{T}$ is an operator that projects transverse to the director

$$
\delta_{i j}^{T}(\mathbf{r})=\delta_{i j}-n_{i}(\mathbf{r}) n_{j}(\mathbf{r}) .
$$

The director field itself has no microscopic definition in terms of the canonical coordinates and momenta of the individual atoms. Its Poisson brackets must therefore be obtained indirectly from those of the alignment vector $\mathbf{P}$. Using the definition of the director given in Eq. (17) and the chain rule of derivatives we can write

$$
\begin{aligned}
\left\{P_{i}(\mathbf{r}), g_{j}\left(\mathbf{r}^{\prime}\right)\right\}= & \rho(\mathbf{r}) S(\mathbf{r})\left\{n_{i}(\mathbf{r}), g_{j}\left(\mathbf{r}^{\prime}\right)\right\} \\
& +n_{i}(\mathbf{r})\left\{\rho(\mathbf{r}) S(\mathbf{r}), g_{j}\left(\mathbf{r}^{\prime}\right)\right\} .
\end{aligned}
$$

Since $n_{i}(\mathbf{r})\left\{n_{i}(\mathbf{r}), \Phi_{\mu}\left(\mathbf{r}^{\prime}\right)\right\}=0$, for any field $\Phi_{\mu}$, we obtain

$$
\left\{n_{i}(\mathbf{r}), g_{j}\left(\mathbf{r}^{\prime}\right)\right\}=\frac{1}{\rho(\mathbf{r}) S(\mathbf{r})} \delta_{i k}^{T}(\mathbf{r})\left\{P_{k}(\mathbf{r}), g_{j}\left(\mathbf{r}^{\prime}\right)\right\} .
$$

\section{HYDRODYNAMIC EQUATIONS FOR THE POLARIZED PHASE}

In this section we derive the hydrodynamic equations in the polarized phase. The order parameter $S(\mathbf{r})$ is assumed to be finite and constant in the following discussion. To calculate the reactive part of the hydrodynamic equations defined 
in Eq. (12) in terms of the Poisson brackets, we need the coarse-grained Hamiltonian for the system

$$
\mathcal{H}[\rho, \mathbf{g}, \mathbf{n}]=\int d^{3} r \frac{\mathbf{g}^{2}(\mathbf{r})}{2 \rho(\mathbf{r})}+F[\rho, \mathbf{n}] .
$$

It consists of a kinetic part and a free energy $F[\rho, \mathbf{n}]$ that depends on the specific system in question and will be specified below.

The nondissipative velocity for the mass-density equation is immediately found to be

$$
V^{\rho}=-\nabla_{i} g_{i}(\mathbf{r}) .
$$

The determination of the nondissipative term for the momentum-density equation is straightforward albeit more tedious. Using Eqs. (23) and (36), we obtain

$$
\begin{aligned}
V_{i}^{\mathbf{g}}= & -\nabla_{j} \frac{g_{i}(\mathbf{r}) g_{j}(\mathbf{r})}{\rho}-\rho(\mathbf{r}) \nabla_{i} \frac{\delta F}{\delta \rho(\mathbf{r})}-n_{k} \nabla_{i}\left[\delta_{j k}^{T} \frac{\delta F}{\delta n_{j}(\mathbf{r})}\right] \\
& +\nabla_{k}\left[n_{k} \delta_{i j}^{T} \frac{\delta F}{\delta n_{i}(\mathbf{r})}\right]-\nabla_{k}\left[\frac{\delta_{j l}^{T} \lambda_{i k l}}{\rho(\mathbf{r}) S(\mathbf{r})} \frac{\delta F}{\delta n_{j}(\mathbf{r})}\right] .
\end{aligned}
$$

Similarly for the director, we obtain using Eq. (36),

$$
V_{i}^{\mathrm{n}}=-v_{j} \boldsymbol{\nabla}_{j} n_{i}+\omega_{i j} n_{j}+\lambda \delta_{i k}^{T} u_{k j} n_{j},
$$

where $v_{i}=\delta \mathcal{H} / \delta g_{i}$ is the velocity and $u_{i j}=\frac{1}{2}\left(\nabla_{i} v_{j}+\nabla_{j} v_{i}\right)$ is the strain-rate tensor, while $\omega_{i j}=\frac{1}{2}\left(\nabla_{i} v_{j}-\nabla_{j} v_{i}\right)$ is the vorticity. Only the part of the tensor $\lambda_{i j k}$ that is transverse to the director enters in the hydrodynamic equations. This is given by

$$
\delta_{j l}^{T}(\mathbf{r}) \lambda_{i k l}(\mathbf{r})=\frac{1-\lambda}{2} \rho(\mathbf{r}) S(\mathbf{r})\left[\delta_{i j}^{T}(\mathbf{r}) n_{k}(\mathbf{r})+\delta_{j k}^{T}(\mathbf{r}) n_{i}(\mathbf{r})\right] .
$$

We now turn our attention to the dissipative part of Eq. (11). Since the mass conservation is exact, there is no dissipative term allowed in the dynamical equation for the mass density. In the case of unbroken Galilean invariance, the time derivative of $\mathbf{g}$ can only couple to the gradients of velocity. As in the nematic case [24], we introduce the tensor of viscosities $\eta_{i j k l}$ and the viscous stress tensor $\sigma_{i j}^{V}$, with the following properties:

$$
\boldsymbol{\nabla}_{j} \eta_{i j k l} \nabla_{k} v_{l}=\nabla_{j} \sigma_{i j}^{V},
$$

where

$$
\begin{aligned}
\sigma_{i j}^{V}= & \alpha_{1} n_{i} n_{j} n_{k} n_{l} u_{k l}+\alpha_{4} u_{i j}+\frac{\alpha_{5}+\alpha_{6}}{2}\left(n_{i} u_{j k}+n_{j} u_{i k}\right) n_{k} \\
& +\rho_{1} \delta_{i j} u_{k k}+\rho_{2}\left(\delta_{i j} n_{k} n_{l} u_{k l}+u_{k k} n_{i} n_{j}\right) .
\end{aligned}
$$

To the lowest order in gradients, there is no difference in the expression of $\sigma_{i j}^{V}$ between the polar and nematic cases. Likewise, as in a nematic liquid crystal, the time derivative $\partial \mathrm{n} / \partial t$ can only couple to a term of the form

$$
-\gamma \delta_{i j}^{T} \frac{\delta F}{\delta n_{j}},
$$

where $\gamma^{-1}$ is a rotational friction.
Thus, collecting the reactive and dissipative terms, we arrive at the following hydrodynamic equations for a liquid crystal with polar orientational order

$$
\begin{gathered}
\frac{\partial \rho}{\partial t}=-\nabla \cdot g(\mathbf{r}), \\
\frac{\partial g_{i}}{\partial t}=-\nabla_{j} \frac{g_{i}(\mathbf{r}) g_{j}(\mathbf{r})}{\rho}-\rho(\mathbf{r}) \nabla_{i} \frac{\delta F}{\delta \rho(\mathbf{r})}+\left(\nabla_{i} n_{j}\right)\left(\frac{\delta F}{\delta n_{j}}\right) \\
+\frac{1+\lambda}{2} \nabla_{k}\left[n_{k} \delta_{i j}^{T} \frac{\delta F}{\delta n_{j}}\right]-\frac{1-\lambda}{2} \nabla_{k}\left[n_{i} \delta_{j k}^{T} \frac{\delta F}{\delta n_{j}}\right]+\nabla_{j} \sigma_{i j}^{V}, \\
\frac{\partial n_{i}}{\partial t}=-v_{j} \nabla_{j} n_{i}-\omega_{i j} n_{j}+\lambda \delta_{i k}^{T} u_{k j} n_{j}-\gamma \delta_{i j}^{T} \frac{\delta F}{\delta n_{j}} .
\end{gathered}
$$

We note that the hydrodynamic equations for the polarized phase have the same form as those for a conventional nematic, as given, for instance, in Ref. [24], although of course with a different microscopic expression for $\lambda$. The only differences between the two sets of equations come from differences in the free energy.

Using the form for the free energy given in Eqs. (20) and (19), the hydrodynamic equations of a polar liquid crystal are given by the continuity equation (45) and

$$
\begin{aligned}
\partial_{t} n_{i}(\mathbf{r})= & -v_{j}(\mathbf{r}) \nabla_{j} n_{i}(\mathbf{r})-\omega_{i j} n_{j} \\
& +\lambda \delta_{i k}^{T} u_{k j} n_{j}+\gamma \delta_{i j}^{T} \frac{B}{\rho_{0}} \nabla_{j} \rho(\mathbf{r})-\gamma \delta_{i j}^{T} \frac{\delta F_{n}}{\delta n_{j}}, \\
\partial_{t} g_{i}(\mathbf{r})= & -\nabla_{j} \frac{g_{i}(\mathbf{r}) g_{j}(\mathbf{r})}{\rho}-B \frac{\rho(\mathbf{r})}{\rho_{0}} \nabla_{i} \nabla_{j} n_{j}(\mathbf{r})-\frac{B}{\rho_{0}}\left(\nabla_{i} n_{j}\right)\left(\nabla_{j} \rho\right) \\
& -\frac{1+\lambda}{2 \rho_{0}} B \nabla_{k}\left[\delta_{i j}^{T} n_{k} \nabla_{j} \rho\right]+\frac{1-\lambda}{2 \rho_{0}} B \nabla_{k}\left[\delta_{j k}^{T} n_{i} \nabla_{j} \rho\right] \\
& -\rho(\mathbf{r}) \nabla_{i} \frac{\delta F_{n}}{\delta \rho(\mathbf{r})}+\left(\nabla_{i} n_{j}\right)\left(\frac{\delta F_{n}}{\delta n_{j}}\right) \\
& +\frac{1+\lambda}{2} \nabla_{k}\left[n_{k} \delta_{i j}^{T} \frac{\delta F_{n}}{\delta n_{j}}\right]-\frac{1-\lambda}{2} \nabla_{k}\left[n_{i} \delta_{j k}^{T} \frac{\delta F_{n}}{\delta n_{j}}\right] \\
& +\nabla_{j} \sigma_{i j}^{V},
\end{aligned}
$$

where

$$
\begin{aligned}
\frac{\delta F_{n}}{\delta \rho}= & C_{1}\left(\frac{\delta \rho}{\rho_{0}^{2}}\right)-C_{2} \boldsymbol{\nabla}^{2} \rho, \\
\frac{\delta F_{n}}{\delta n_{j}}= & -K_{1} \boldsymbol{\nabla}_{j}(\boldsymbol{\nabla} \cdot \mathbf{n})+K_{2}[\boldsymbol{\nabla} \times(\boldsymbol{\nabla} \times \mathbf{n})]_{j} \\
& -\left(K_{2}-K_{3}\right) n_{s}\left(\boldsymbol{\nabla}_{s} n_{k}\right)\left(\nabla_{j} n_{k}\right) \\
& +\left(K_{2}-K_{3}\right) \boldsymbol{\nabla}_{b}\left[n_{b} n_{s}\left(\nabla_{s} n_{j}\right)\right] .
\end{aligned}
$$

The terms in the hydrodynamic equations that are unique to a fluid with macroscopic polar order are those proportional to the elastic constant $B$. These are forbidden by symmetry in the hydrodynamic equations of a nematic. 


\section{DYNAMICS OF THE ALIGNMENT VECTOR}

In the more general case where $S(\mathbf{r})$ is not a constant in Eq. (17), we need to consider the full order parameter of the alignment vector $\mathbf{P}$. The hydrodynamic equations for the alignment vector $\mathbf{P}$ are

$$
\begin{gathered}
\frac{\partial}{\partial t} \rho(\mathbf{r})=-\nabla \cdot \mathbf{g} \\
\frac{\partial}{\partial t} P_{i}(\mathbf{r})=-v_{j}(\mathbf{r}) \nabla_{j} P_{i}(\mathbf{r})+P_{j}(\mathbf{r}) \nabla_{j} v_{i}(\mathbf{r}) \\
-P_{i}(\mathbf{r})(\boldsymbol{\nabla} \cdot \mathbf{v})-\lambda_{i j k} \boldsymbol{\nabla}_{k} v_{j}(\mathbf{r})-\gamma^{\prime} \frac{\delta F}{\delta P_{i}}, \\
\frac{\partial}{\partial t} g_{i}(\mathbf{r})=-\nabla_{j} \frac{g_{i}(\mathbf{r}) g_{j}(\mathbf{r})}{\rho}-\rho(\mathbf{r}) \boldsymbol{\nabla}_{i} \frac{\delta F}{\delta \rho(\mathbf{r})} \\
+\nabla_{k}\left[P_{k}(\mathbf{r}) \frac{\delta F}{\delta P_{i}(\mathbf{r})}\right]-P_{j}\left(\mathbf{r}\left[\nabla_{i} \frac{\delta F}{\delta P_{j}(\mathbf{r})}\right]\right) \\
-\nabla_{k}\left[\lambda_{i j k} \frac{\delta F}{\delta P_{j}(\mathbf{r})}\right]-\nabla_{j} \sigma_{i j}^{V} .
\end{gathered}
$$

The equation for the director can be obtained from Eq. (53) by assuming $S=$ constant and using the relation

$$
\partial_{t} n_{i}(\mathbf{r})=\frac{1}{\rho(\mathbf{r}) S(\mathbf{r})} \delta_{i k}^{T}(\mathbf{r}) \partial_{t} P_{k}(\mathbf{r}) .
$$

\section{HYDRODYNAMIC MODES IN THE POLARIZED PHASE}

In the isotropic phase the only hydrodynamic fields are the four conserved densities: number density and three components of the momentum (energy fluctuations are not considered here). The corresponding four hydrodynamic modes are those of a conventional isotropic liquid: two propagating sound wave describing the decay of density and longitudinal momentum fluctuations, and two diffusive shear modes, describing the decay of the two tranverse components of the momentum density. We now consider the long wavelength, low frequency dynamics in a uniformly polarized phase, characterized by uniform equilibrium values $\rho_{0}$ of the density $\mathbf{v}_{0}=0$ of the flow velocity and $\mathbf{n}_{0}=\hat{\mathbf{z}}$ of the director field. In order to evaluate the hydrodynamic modes of the polarized state, we expand the hydrodynamic equations to linear order in the fluctuations of density $\delta \rho=\rho-\rho_{0}$, momentum $\delta \mathbf{g}$ $\simeq \rho_{0} \mathbf{v}$, and director $\delta \mathbf{n}=\mathbf{n}-\hat{\mathbf{z}}$ about their equilibrium values (to lowest order $\hat{\mathbf{z}} \cdot \delta \mathbf{n}=0$ ). We will ignore any variation in temperature in our consideration. We consider wavelike fluctuations whose space and time dependence is of the form $\exp [i \mathbf{q} \cdot \mathbf{r}-i \omega t]$ and choose the coordinate system so that the wave vector $\mathbf{q}$ lies in the $(x z)$ plane, i.e., $\mathbf{q}=\left(q_{x}, q_{z}\right)$ and $q_{y}$ $=0$. We then project velocity and director fluctuations along the axes of an orthogonal coordinate system defined by the unit vectors $\left(\hat{\mathbf{z}}, \hat{\mathbf{q}}_{x}, \hat{\mathbf{z}} \times \hat{\mathbf{q}}_{x}\right)$, with $\hat{\mathbf{q}}_{x}=q_{x} / q$. The equations for velocity and director fluctuations transverse to both the direction of polarization $\hat{\mathbf{z}}$ and $\hat{\mathbf{q}}_{x}$ defined as

$$
\begin{aligned}
v_{\mathbf{q}}^{y} & =\left(\hat{\mathbf{z}} \times \hat{\mathbf{q}}_{x}\right) \cdot \mathbf{v}_{q}, \\
\delta n_{\mathbf{q}}^{y} & =\left(\hat{\mathbf{z}} \times \hat{\mathbf{q}}_{x}\right) \cdot \delta \mathbf{n}_{q},
\end{aligned}
$$

decouple from the others and are given by

$$
\begin{aligned}
\partial_{t} \delta n_{\mathbf{q}}^{y} & =-\gamma K_{T}(\hat{\mathbf{q}}) q^{2} \delta n_{q_{y}}-i \frac{1-\lambda}{2} q_{z} v_{\mathbf{q}}^{y}, \\
\partial_{t} v_{\mathbf{q}}^{y} & =-\nu_{T}(\hat{\mathbf{q}}) q^{2} v_{q_{y}}+i \frac{1+\lambda}{2 \rho_{0}} K_{T}(\hat{\mathbf{q}}) q^{2} q_{z} \delta n_{\mathbf{q}}^{y},
\end{aligned}
$$

where $\hat{\mathbf{q}}=\left(q_{x} / q, q_{z} / q\right)$ and

$$
\begin{aligned}
& K_{T}(\hat{\mathbf{q}})=\left[K_{2} q_{x}^{2}+K_{3} q_{z}^{2}\right] / q^{2}, \\
& \nu_{T}(\hat{\mathbf{q}})=\frac{\alpha_{4}}{2 \rho_{0}}+\frac{\alpha_{5}+\alpha_{6}}{4 \rho_{0}} \frac{q_{z}^{2}}{q^{2}} .
\end{aligned}
$$

The transverse eigenvalues are identical to those of nematic liquid crystals and are given by

$$
\begin{aligned}
i \omega_{ \pm}^{T}= & \frac{q^{2}}{2}\left[\nu_{T}(\hat{\mathbf{q}})+\gamma K_{T}(\hat{\mathbf{q}})\right] \\
& \pm \frac{q^{2}}{2} \sqrt{\left[\nu_{T}(\hat{\mathbf{q}})-\gamma K_{T}(\hat{\mathbf{q}})\right]^{2}+\hat{q}_{z}^{2} \frac{1-\lambda^{2}}{\rho_{0}} K_{T}(\hat{\mathbf{q}}) .}
\end{aligned}
$$

These modes are always diffusive and describe the coupled decay of vorticity and twist fluctuations. For $q_{z}=0$ the transverse modes decouple and are simply given by

$$
\begin{aligned}
& i \omega_{n}^{T}=\gamma K_{2} q_{x}^{2}, \\
& i \omega_{v}^{T}=\frac{\alpha_{4}}{2 \rho_{0}} q_{x}^{2},
\end{aligned}
$$

where $\omega_{n}^{T}$ describes the diffusion of fluctuations in the local twist and $\omega_{v}^{T}$ controls the decay of fluctuations in the vorticity.

The remaining four hydrodynamic fluctuations, describing the dynamics of density $\delta \rho_{\mathrm{q}}$, velocity and director components longitudinal to $\hat{\mathbf{q}}_{x}, v_{\mathbf{q}}^{x}=\hat{\mathbf{q}}_{x} \cdot \mathbf{v}_{q}$ and $\delta n_{q}^{x}=\hat{\mathbf{q}}_{x} \cdot \delta \mathbf{n}_{q}$, and velocity component along the direction of polarization, $v_{\mathbf{q}}^{z}$ are coupled. The linearized equations are given by

$$
\begin{aligned}
\partial_{t} \delta \rho_{\mathbf{q}}= & -i \rho_{0} q_{x} v_{\mathbf{q}}^{x}-i \rho_{0} q_{z} v_{\mathbf{q}}^{z}, \\
\partial_{t} \delta n_{\mathbf{q}}^{x}= & -\gamma K_{L}(\hat{\mathbf{q}}) q^{2} \delta n_{\mathbf{q}}^{x}+i \gamma \frac{B}{\rho_{0}} q_{x} \delta \rho_{\mathbf{q}} \\
& -i \frac{1-\lambda}{2} q_{z} v_{\mathbf{q}}^{x}+i \frac{1+\lambda}{2} q_{x} v_{\mathbf{q}}^{z}, \\
\partial_{t} v_{\mathbf{q}}^{x}= & -\nu_{L}(\hat{\mathbf{q}}) q^{2} v_{\mathbf{q}}^{x}-\nu_{L}^{\prime} q_{x} q_{z} v_{\mathbf{q}}^{z}-i q_{x} \frac{C_{1}}{\rho_{0}^{2}} \delta \rho_{\mathbf{q}} \\
& +\frac{1+\lambda}{2 \rho_{0}^{2}} B q_{z} q_{x} \delta \rho_{\mathbf{q}} \\
& +i \frac{1+\lambda}{2 \rho_{0}} K_{L}(\hat{\mathbf{q}}) q_{z} q^{2} \delta n_{\mathbf{q}}^{x},
\end{aligned}
$$




$$
\begin{aligned}
\partial_{t} v_{\mathbf{q}}^{z}= & -\nu_{z}(\hat{\mathbf{q}}) q^{2} v_{\mathbf{q}}^{z}-\nu_{L}^{\prime} q_{x} q_{z} v_{\mathbf{q}}^{x}-i q_{z} \frac{C_{1}}{\rho_{0}^{2}} \delta \rho_{\mathbf{q}} \\
& -\frac{1-\lambda}{2 \rho_{0}^{2}} B q_{x}^{2} \delta \rho_{\mathbf{q}} \\
& -i \frac{1-\lambda}{2 \rho_{0}} K_{L}(\hat{\mathbf{q}}) q_{x} q^{2} \delta n_{\mathbf{q}}^{x}
\end{aligned}
$$

where

$$
\begin{aligned}
K_{L}(\hat{\mathbf{q}})= & {\left[K_{1} q_{x}^{2}+K_{3} q_{z}^{2}\right] / q^{2}, } \\
\nu_{L}(\hat{\mathbf{q}})= & \frac{\alpha_{4}+\rho_{1}}{\rho_{0}} \frac{q_{x}^{2}}{q^{2}}+\frac{2 \alpha_{4}+\alpha_{5}+\alpha_{6}}{4 \rho_{0}} \frac{q_{z}^{2}}{q^{2}}, \\
\nu_{z}(\hat{\mathbf{q}})= & \frac{\alpha_{1}+\alpha_{4}+\alpha_{5}+\alpha_{6}+\rho_{1}+2 \rho_{2}}{\rho_{0}} \frac{q_{z}^{2}}{q^{2}} \\
& +\frac{2 \alpha_{4}+\alpha_{5}+\alpha_{6}}{4 \rho_{0}} \frac{q_{x}^{2}}{q^{2}}, \\
\nu_{L}^{\prime}= & \frac{\rho_{1}+\rho_{2}}{\rho_{0}}+\frac{2 \alpha_{4}+\alpha_{5}+\alpha_{6}}{4 \rho_{0}} .
\end{aligned}
$$

The corresponding hydrodynamic equations for a nematic liquid crystal are obtained from Eqs. (65)-(68) by simply setting $B=0$. For comparison we recall that there are four longitudinal hydrodynamic modes in a compressible nematic liquid crystal: two propagating sound waves and two diffusive modes describing the coupled decay of splay and velocity fluctuations [28]. The same four modes are present in a polarized liquid crystal, but their decay rates are modified in important ways by the new symmetry term breaking term proportional to $B$.

We calculate the hydrodynamic frequencies up to $o\left(q^{2}\right)$ by solving the eigenvalue problem perturbatively. As expected, we find two propagating and two purely diffusive modes. The two propagating eigenmodes are the sound waves of the fluid and are given by

$$
i \omega_{ \pm}^{s} \approx \pm i \sqrt{\frac{C_{1}}{\rho_{0}}} q+\frac{q^{2}}{2}\left[\nu_{L} \hat{q}_{x}^{2}+\nu_{z} \hat{q}_{z}^{2}+2 \nu_{L}^{\prime} \hat{q}_{x}^{2} \hat{q}_{z}^{2}+\frac{\gamma B^{2}}{C_{1}} \hat{q}_{x}^{2}\right] .
$$

To lowest order in the wave vector the propagation speed is proportional to $\sqrt{\frac{\bar{C}_{1}}{\rho_{0}}}$. As in isotropic liquids, it is determined entirely by the compressional modulus $C_{1}$ of the fluid and receive corrections proportional to $B$. The coupling to polar order described by the elastic constant $B$ increases the relaxation rate, but has no qualitative effect on the structure of the sound waves. In the incompressible limit, $C_{1}$ tends to infinity and all contributions to the dynamics of the system from terms proportional to $B$ vanish. This is evident in Eq. (50) as the $\mathcal{Z}_{2}$-odd term becomes a surface term.
The eigenfrequencies of the two diffusive modes are given by

$$
\begin{aligned}
i \omega_{ \pm}^{\mathrm{diff}}= & q^{2}\left[\frac{\gamma K(\hat{\mathbf{q}})+\mu(\hat{\mathbf{q}})}{2}\right. \\
& \left. \pm \frac{1}{2} \sqrt{[\gamma K(\hat{\mathbf{q}})-\mu(\hat{\mathbf{q}})]^{2}+\frac{K(\hat{\mathbf{q}})}{\rho_{0}}\left[1-\lambda^{2}\left(\hat{q}_{x}^{2}-\hat{q}_{z}^{2}\right)^{2}\right]}\right],
\end{aligned}
$$

where

$$
\begin{aligned}
K(\hat{\mathbf{q}}) & =K_{L}(\hat{\mathbf{q}})-\frac{B^{2}}{C_{1}} \hat{q}_{x}^{2}, \\
\mu(\hat{\mathbf{q}}) & =\nu_{z}(\hat{\mathbf{q}}) \hat{q}_{x}^{2}+\nu_{L}(\hat{\mathbf{q}}) \hat{q}_{z}^{2}-2 \nu_{L}^{\prime} \hat{q}_{x}^{2} \hat{q}_{z}^{2} \\
& =\left[2 \alpha_{4}+\alpha_{5}+\alpha_{6}+4 \alpha_{1} \hat{q}_{x}^{2} \hat{q}_{z}^{2}\right] / 4 \rho_{0} .
\end{aligned}
$$

When $K(\hat{\mathbf{q}})=0$, the diffusive modes become unstable. This is precisely the splay instability discussed in Sec. III by examining the free energy of the system.

In conclusion, we have derived the dynamical equations for the director $\mathbf{n}$ and the alignment vector $\mathbf{P}$, starting from the microscopic expressions of the conserved quantities and carrying out rigorous coarse graining via the Poisson-bracket approach. Our results are consistent with the equations for nematic liquid crystals derived earlier using similar approaches $[23,24]$. Furthermore, we have investigated the hydrodynamic modes of an ordered phase with macroscopic polarization. We have found that the polarity of the director field introduces a frustration in the system. As a result, a state of uniform local density and director orientation can become unstable to the growth of long wavelength splay fluctuations in a region of parameters. The splay instability arises solely from the addition of $\mathcal{Z}_{2}$-odd terms in the free energy, which are generically allowed on the basis of symmetry considerations in any polar liquid. We therefore believe that the existence of the instability is a general feature of any polar fluid, regardless of the origin of the polarity.

It is our hope that the results reported in this paper will be useful in providing a rigorous justification of the various equations used previously in studying the ferroelectric phase of polar liquid crystals. It is our further hope that our results, presented here in the context of equilibrium physics, will also serve as a starting point in understanding the behavior of analogous systems in the realm of nonequilibrium physics where the polarity of these nematic phases is driven by active mechanisms.

\section{ACKNOWLEDGMENTS}

K.S. acknowledges valuable discussions with John Toner. W.K. and M.C.M. were supported by NSF Grants Nos. DMR-0219292 and DMR-0305407. 
[1] P. M. Chaikin and T. C. Lubensky, Principles of Condensed Matter Physics (Cambridge University Press, New York, 1980).

[2] B. A. DiDonna and R. D. Kamien, Phys. Rev. Lett. 89, 215504 (2002); E. Grelet et al., ibid. 86, 3791 (2001); B. Pansu et al., ibid. 62, 658 (2000); E. Grelet, B. Pansu, and H. T. Nguyen, Phys. Rev. E 64, 010703(R) (2001).

[3] T. C. Lubensky and L. Radzihovsky, Phys. Rev. E 66, 031704 (2002); T. Niori, T. Sekine, J. Watanabe, T. Furukawa, and H. Takezoe, J. Mater. Chem. 6, 1231 (1996); D. R. Link, G. Natale, R. Shao, J. E. Maclennan, N. A. Clark, E. Korblova, and D. M. Walba, Science 278, 1924 (1997); D. M. Walba, E. Korblova, R. Shao, J. E. Maclennan, D. R. Link, M. A. Glaser, and N. A. Clark, ibid. 288, 2181 (2000).

[4] R. B. Meyer, L. Liebert, L. Strzelecki, and P. Keller, J. Phys. (France) Lett. 36, L69 (1975).

[5] A. D. L. Chandani, Y. Ouchi, H. Takezoe, A. Fukuda, K. Terashima, K. Furukawa, and A. Kishi, Jpn. J. Appl. Phys., Part 2 28, L1261 (1989).

[6] T. Watanabe, S. Miyata, T. Furukawa, H. Takezoe, T. Nishi, M. Sone, A. Migita, and J. Watanabe, Jpn. J. Appl. Phys., Part 2 35, L505 (1996).

[7] T. Furukawa, K. Ishikawa, H. Takezoe, A. Fukuda, T. Watanabe, S. Miyata, T. Nishi, M. Sone, and J. Watanabe, Nonlinear Opt. 15, 167 (1996).

[8] T. Furukawa, H. Takezoe, T. Nishi, T. Mitsukuchi, A. Migita, J. Watanabe, T. Watanabe, and S. Miyata, Mol. Cryst. Liq. Cryst. Sci. Technol., Sect. A 299, 105 (1997).

[9] H. Takezoe and J. Watanabe, Mol. Cryst. Liq. Cryst. Sci. Technol., Sect. A 328, 325 (1999).

[10] J. Watanabe, L. Yuqing, H. Tuchiya and H. Takezoe, Mol. Cryst. Liq. Cryst. Sci. Technol., Sect. A 346, 9 (2000).

[11] B. Mettout, P. Tolédano, H. Takezoe, and J. Watanabe, Phys. Rev. E 66, 031701 (2002).
[12] J. Lee and S. D. Lee, Mol. Cryst. Liq. Cryst. Sci. Technol., Sect. A 254, 395 (1994).

[13] M. Baus and J. L. Colot, Phys. Rev. A 40, 5444 (1989).

[14] E. M. Terentjev, M. A. Osipov, and T. J. Sluckin, J. Phys. A 27, 7047 (1994).

[15] B. Park, J. W. Wu, and H. Takezoe, Phys. Rev. E 63, 021707 (2001).

[16] P. Palffy-Muhoray, M. A. Lee, and R. G. Petschek, Phys. Rev. Lett. 60, 2303 (1988).

[17] D. Wei and G. N. Patey, Phys. Rev. Lett. 68, 2043 (1992).

[18] P. I. C. Teixeira, J. M. Tavares, and M. M. Telo da Gama, J. Phys.: Condens. Matter 12, R411 (2000)

[19] F. Biscarini, C. Zannoni, C. Chiccoli, and P. Pasini, Mol. Phys. 73, 439 (1991)

[20] J. J. Weiss, D. Levesque, and G. J. Zarragoicoechea, Phys. Rev. Lett. 69, 913 (1993)

[21] We should note that this is in contrast to molecules forming a smectic- $C^{*}$ phase or in banana molecules, the dipole moment usually has a component perpendicular to the molecular axis.

[22] P. G. De Gennes and J. Prost, The Physics of Liquid Crystals (Oxford University Press, New York, 1995), Sec. 1.3.2, p. 12; N. Boccara, Ann. Phys. (N.Y.) 76, 72 (1973).

[23] R. D. Kamien, Phys. Rev. E 61, 2888 (2000).

[24] H. Stark and T. C. Lubensky, Phys. Rev. E 67, 061709 (2003).

[25] R. Zwanzig, Phys. Rev. 124, 983 (1961).

[26] K. Kawasaki, Ann. Phys. (N.Y.) 61, 1 (1970).

[27] H. Mori, H. Fujisaka, and H. Shigematsu, Prog. Theor. Phys. 51, 109 (1973).

[28] M. J. Stephen and J. P. Straley, Rev. Mod. Phys. 46, 617 (1974).

[29] W. I. Minkin, O. A. Osipov, and Y. A. Zhdanov, Dipole Moments in Organic Chemistry (Plenum Press, New York, 1970).

[30] G. E. Volovik, Ann. Phys. (N.Y.) 125, 67 (1980); G. E. Volovik, JETP Lett. 31, 6273 (1980); 